Psychotherapeut 2020 $65: 277-284$

https://doi.org/10.1007/s00278-020-00433-x

Online publiziert: 27. Mai 2020

(c) Der/die Autor(en) 2020
Bernhard Strauß

Institut für Psychosoziale Medizin und Psychotherapie, Universitätsklinikum Jena, Jena, Deutschland

\title{
Instrumente und Materialien zur Unterstützung gruppenpsychotherapeutischer Praxis
}

\begin{abstract}
Die Gesundheitspolitik verfolgt die erklärte Absicht, die Attraktivität der Durchführung von Gruppen im ambulanten Rahmen zu steigern. Hierzu wurden in den letzten Jahren strukturbildende Maßnahmen ergriffen, die vermutlich dazu beitragen, dass zunehmend ambulante Gruppenbehandlungen angeboten werden. Danach befragt, wie gruppenpsychotherapeutisch Tätige zu ihren Kompetenzen gekommen sind (Strauß et al. 2015), war die am häufigsten genannten Option das "Learning by doing" oder alternativ die Beobachtung erfahrenerer Gruppentherapeuten. Eine wirklich gezielte Erarbeitung gruppentherapeutischer Kompetenz unter Anleitung mit Vermittlung von Theorie, praktischer Reflexion und Supervision dürfte bislang noch immer die Ausnahme sein.
\end{abstract}

\section{Förderung der Gruppentherapie}

Die erklärte Absicht der Gesundheitspolitik, die Attraktivität der Durchführung von Gruppen im ambulanten Rahmen zu steigern, wurde von der letzten Bundesregierung 2014 in ihrem Koalitionsver- trag formuliert ${ }^{1}$. Nach einer Erhebung der Bundespsychotherapeutenkammer (BPtK 2018) zur Wirkung der Reform der Psychotherapierichtlinien, die sich auf die Jahre 2016 und 2017 bezog, war ein Effekt bezüglich der Gruppentherapie noch nicht wirklich nachweisbar.

Es wurde schon vorher immer wieder berichtet, dass die große Mehrheit ambulant tätiger Psychotherapeuten trotz vorliegender Abrechnungsgenehmigung (ca. 30\%) und damit verbundener Qualifikation keine Gruppentherapien durchführt. Der Bericht über die BPtK-Studie konstatierte: „Nach Ansicht der befragten Psychotherapeuten haben sich durch die Reform der Psychotherapierichtlinie die Grundlagen für ein ambulantes gruppentherapeutisches Angebot nicht wesentlich verbessert. Fast alle Psychotherapeuten (91,6\%), die über eine Abrechnungsgenehmigung für Gruppenpsychotherapie verfügen und bisher keinen $\mathrm{Ge}$ brauch hiervon gemacht haben, tun dies auch nach der Reform nicht. Auch die Psychotherapeuten, die vor der Reform bereits Gruppenpsychotherapien angeboten haben, weiteten ihre gruppenpsy-

\footnotetext{
1 "Wir wollen in der psychotherapeutischen Versorgung Wartezeiten reduzieren und mehr Betroffenen ein zeitnahes Angebot für Kurzzeittherapie eröffnen. Hierzu werden wir das Antrags- und Gutachterverfahren entbürokratisieren, die Gruppentherapie fördern und den Gemeinsamen Bundesausschuss beauftragen, in einer gesetzlich definierten Frist die Psychotherapierichtlinie zu überarbeiten" (Koalitionsvertrag von CDU, CSU und SPD, "Deutschlands Zukunft gestalten", 2014, S. 54).
}

chotherapeutischen Angebote kaum aus. Lediglich 16,3\% der Psychotherapeuten, die bereits zuvor Gruppenpsychotherapie durchgeführt haben, haben ihr Angebot ausgebaut" (S. 27).

Seither sind allerdings weitere strukturbildende Maßnahmen ergriffen worden, wie beispielsweise der sofortige Wegfall der Antragspflicht für Gruppentherapien, die im Verbund mit den anderen Maßnahmen (bessere Kombinierbarkeit von Einzel- und Gruppentherapie, bessere Vergütung, Behandlungsbeginn in kleineren Gruppen etc.) vermutlich nun doch dazu beitragen, dass niedergelassene Psychotherapeutinnen und Psychotherapeuten zunehmend auch Gruppenbehandlungen anbieten werden. Zumindest im Supervisionskontext ist zu bemerken, dass Gruppen attraktiver und damit auch häufiger angewandt werden.

Vor diesem Hintergrund stellt sich die Frage, wie gruppenpsychotherapeutisch Tätige unterstützt werden können, durch wissenschaftlich fundiertes Material, das der Strukturierung, der besseren Durchführung und v.a. der Evaluation von Gruppentherapie dienen kann.

\section{"CORE battery“}

Die Idee, ein „Optimierungs-Toolkit“ für die Auswahl von Gruppenpatientinnen und Gruppenpatienten, die Unterstützung der Prozessbeobachtung und die Ergebnisbewertung von Gruppen zu erstellen, wurde erstmals in den 1980erJahren durch die American Group Psy- 
Tab. 1 Verschiedene Felder gruppentherapeutischer Praxis sowie dazu gehörige Instrumente und Materialien („IMELDA“)

\begin{tabular}{|c|c|c|}
\hline I & Information und Vorbereitung & $\begin{array}{l}\text { Handouts, Flyer, Verträge (vgl. Burlingame et al. } \\
\text { 2006) }\end{array}$ \\
\hline M & Motivation und Auswahl & $\begin{array}{l}\text { Group Therapy Questionnaire (GTQ), Group Readi- } \\
\text { ness Questionnaire (GRQ) }\end{array}$ \\
\hline$E$ & $\begin{array}{l}\text { Evaluation und Wirksamkeits- } \\
\text { messung }\end{array}$ & $\begin{array}{l}\text { Outcome Questionnaire (OQ-45, Y-OQ), Inventar } \\
\text { zur Erfassung interpersonaler Probleme (IIP), Beck- } \\
\text { Depressions-Inventar (BDI), Brief Symptom Inventory } \\
\text { (BSI) ... } \\
\text { Fragebogen zu Nebenwirkungen in Gruppenpsycho- } \\
\text { therapien und unerwünschten Gruppenerfahrungen } \\
\text { (NUGE) }\end{array}$ \\
\hline $\mathrm{L}$ & Leiterstil und Leiterverhalten & $\begin{array}{l}\text { Group Psychotherapy Intervention Rating Scale } \\
\text { (GPIRS) }\end{array}$ \\
\hline D & Dokumentation & $\begin{array}{l}\text { Operationalisierte Basisdokumentation von Grup- } \\
\text { penpsychotherapien (OBG) }\end{array}$ \\
\hline$A$ & Allianz und Prozessmerkmale & $\begin{array}{l}\text { Gruppenfragebogen (GQ), Gruppenklima-Fragebo- } \\
\text { gen (GCQ), Kohäsionsskala des Therapeutic Factors } \\
\text { Inventory (TFI) ... }\end{array}$ \\
\hline
\end{tabular}

chotherapy Association (AGPA) realisiert. Es wurde eine "CORE battery“ erstellt, die in erster Linie Materialien enthalten waren, mit denen Gruppentherapeuten die Wirksamkeit ihrer Tätigkeit auf der Basis von Patientenurteilen und Selbsteinschätzungen bewerten sollten. Das Kürzel CORE stand damals für „clinical outcome results“. Diese Materialsammlung war entsprechend primär am Therapieergebnis orientiert (MacKenzie und Dies 1982). Bezogen auf gruppenspezifische Instrumente, die zur Erfassung von Prozess und Ergebnis von Gruppentherapien dienen, kann als ein gewisses Äquivalent die Sammlung von Instrumenten gelten, die von Strauß et al. (1996) publiziert wurde.

\section{"IMELDA"}

Im Jahr 2003 beauftragte der Vorstand der AGPA eine neu gebildete Task Force (unter der Leitung von G.M. Burlingame und B. Strauß), eine erweiterte CORE battery $\mathrm{zu}$ entwickeln. Diese sollte neben einer Sammlung von ergebnisorientierten Instrumenten Materialien zu Auswahl und Vorbereitung von Patientinnen und Patienten sowie Prozessmaße, die verschiedene Aspekte von Gruppenmerkmalen erfassen, enthalten. Diese Erweiterung spiegelte schlicht die Weiterentwicklung der Forschung in der Gruppenpsychotherapie und spezifische Bedürfnisse von
Praktikern, die innerhalb der AGPA die Mehrheit bilden.

In Form eines Manuals wurde das Ergebnis der Task Force 2006 in englischer Sprache publiziert (Burlingame et al. 2006; Strauß et al. 2008). Mittlerweile wurde das zugrunde gelegte Modell der CORE-Gruppe, in der v. a. die Information und Vorbereitung, die Überprüfung der Motivation und Auswahl, die Evaluation der Gruppenbehandlungen und der Fokus auf Prozessmerkmale und Allianz im Vordergrund standen, erweitert. Insbesondere wurden die beiden Dimensionen „Leiterverhalten“ und „Leiterstil“ sowie die Dokumentation von Gruppensitzungen bzw. deren Verläufen in das Modell aufgenommen, das in $\bullet$ Tab. 1 unter Verwendung des Akronyms IMELDA dargestellt ist. Die Materialien sind naturgemäß auch für die Gruppenpsychotherapieforschung geeignet, die Idee von CORE bzw. IMELDA ist es aber, speziell Praktikern die Möglichkeit einer kontinuierlichen Erfassung von Prozessmerkmalen und Veränderungen aus der Sicht einzelner Gruppenmitglieder zu geben.

Im Folgenden werden die einzelnen Komponenten von IMELDA, auch unter Berücksichtigung der CORE battery, beispielhaft beschrieben, mit Verweisen auf einzelne Instrumente, die Gruppentherapeutinnen und Gruppentherapeuten bei der Durchführung und der Reflexion der eigenen Arbeit nützlich sein können.

\section{I: Information und Vorbereitung}

In Fort- und Weiterbildungsveranstaltungen zur Gruppenpsychotherapie nennen Teilnehmerinnen und Teilnehmer auf die Frage, welche Bedenken und Befürchtungen Patienten gegenüber Gruppen und Gruppentherapien äußern, immer wieder begrenzte und ähnliche Zahlen der Themen, die sich wie im Folgenden zusammenfassen lassen.

Vertraulichkeit und mangelndes Vertrauen. Die Befürchtung, dass intime Informationen aus Gruppensitzungen geraten und anderen Personen im Umkreis einzelner Patienten bekannt werden, ist weit verbreitet und hält sich.

\section{Generelle Angst vor anderen Men-}

schen. Angst und andere Befürchtungen speisen sich häufig aus negativen interpersonalen Erfahrungen, die einzelne Patienten im Laufe ihrer Sozialisation gesammelt haben und die oft schon im Kindesalter begannen.

Befürchtung vor Kontrollverlust. Es ist Gruppen quasi immanent, dass ihre „Dynamik“ nicht $100 \%$ ig vorhersag- und kontrollierbar ist. Besonders ängstliche Menschen sehen hierin einen Grund, sich von Gruppen fernzuhalten, da sie unerwartete Ereignisse sowie den Verlust der Kontrolle über die eigenen Emotionen und Verhaltensweisen fürchten.

Zweifel am Erfolg von Gruppen. In den Köpfen vieler Patienten, (beachte) aber auch Psychotherapeuten gelten Gruppeninterventionen immer noch als eine Behandlung 2. Wahl, was angesichts der überzeugenden Evidenz natürlich einfach entkräftet werden kann (Strauß et al. im vorliegenden Heft).

\section{Negative Vorerfahrung mit Gruppen.}

Viele Menschen haben im Laufe des Lebens speziell mit Gruppen (meist nichttherapeutischen Gruppen, z. B. im Ausbildungs- oder Berufskontext) negative Erfahrungen gemacht. Diese halten sie davon ab, Gruppeninterventionen auch nur im Ansatz als therapeutisches Medium für hilfreich zu halten. 
Scham und Probleme mit Selbstöffnung. Es ist vermutlich ganz natürlich, dass die Vorstellung, sich gegenüber mehr oder weniger unbekannten Anderen öffnen zu müssen und über eigene, oft schambesetzte Themen zu sprechen, zunächst einmal ängstigt.

Belastung durch andere und Angst vor Retraumatisierung. Viele Patienten befürchten, dass sie zu den eigenen Problemen noch die Last der Probleme anderer Patienten aus einer Gruppe mitübernehmen müssen, bzw. dass Themen, die andere in Gruppen äußern, eigene traumatische Erfahrungen reaktivieren könnten.

Angst vor Unbekannten. Dies ist ein weiterer Grund für die Vorsicht und Angst vieler Patienten gegenüber Gruppen. Erfahrungsgemäß können sich Psychotherapeuten oftmals gar nicht vorstellen, dass Menschen mit anderer Sozialisation, die also z.B. keine Selbsterfahrungsgruppen erlebt haben, oft völlig diffuse Vorstellungen davon haben, was in einer therapeutischen Gruppe überhaupt passieren kann und soll.

Sicher gibt es noch weitere Bedenken, die Patienten ins Feld führen, wenn ihnen die Indikation zur Gruppenpsychotherapie gestellt wird. Die oben genannten werden aber fast immer als Erfahrung von gruppentherapeutisch Tätigen genannt und machen deutlich, dass es wichtig ist, die beschriebenen Vorbehalte und Befürchtungen vor der endgültigen Indikationsstellung und Aufnahme von Gruppenmitgliedern aufzugreifen.

Um die Potenziale therapeutischer Gruppen besser nutzen zu können, sind die Vorbereitung und Information der Gruppenmitglieder von essenzieller Bedeutung. Tatsächlich liegt für die Vorhersage von Behandlungsergebnissen bei adäquater Information und Vorbereitung schon lange eine überzeugende empirische Evidenz vor (Burlingame et al. 2004). Vor allem in den letzten Jahrzehnten des letzten Jahrhunderts wurden viele Studien durchgeführt, deren Ergebnisse zeigen, dass eine Gruppenvorbereitung, ein „pregroup training“, die Effektivität von Gruppen sowohl auf individueller wie auch auf Gruppenebene steigern

Psychotherapeut 2020 -65:277-284 https://doi.org/10.1007/s00278-020-00433-x

(c) Der/die Autor(en) 2020

B. Strauß

\section{Instrumente und Materialien zur Unterstützung gruppenpsychotherapeutischer Praxis}

\section{Zusammenfassung}

Unter Verwendung des Akronyms IMELDA fasst diese Arbeit eine Auswahl von Instrumenten und Materialien zusammen, die in der Praxis der Gruppenpsychotherapie hilfreich und nützlich sein können. Das Akronym fasst die Aspekte der Information, der Motivation und Auswahl, der Evaluation zusammen sowie das Gruppen/eiterverhalten, die Dokumentation von Gruppensitzungen sowie die Erfassung von Allianz und anderen Prozessmerkmalen. Die verschiedenen Bereiche von "IMELDA" werden skizziert, unter Darstellung beispielhafter Methoden sowie ihrer Qualität und Verfügbarkeit. Insbesondere vor dem Hintergrund der erhofften Zunahme gruppentherapeutischer Angebote auch im ambulanten Kontext ist davon auszugehen, dass eine gewisse Standardisierung von Abläufen und Begleitdiagnostiken in der Gruppenpsychotherapie für Praktikerinnen und Praktiker von Nutzen ist.

Schlüsselwörter

Auswahl · Vorbereitung · Evaluation . Dokumentation - Therapeutische Allianz

\section{Instruments and materials to support the practice of group psychotherapy}

\begin{abstract}
Using the acronym IMELDA, this article summarizes a selection of instruments and materials that can be useful and helpful in the practice of group psychotherapy. The acronym relates to the aspects of information, motivation and selection, evaluation, leader behavior, documentation and the assessment of alliance and other process variables. The various areas of IMELDA are described and illustrated with selected methods as well as
\end{abstract}

their qualities and availability. In view of an expected increase of group treatment options also in the outpatient sector, it is assumed that a certain standardization of procedures and accompanying diagnostics in group psychotherapy could help practitioners.

Keywords

Selection - Preparation - Evaluation .

Documentation - Therapeutic alliance kann. Im deutschsprachigen Raum wurde dieses Thema vor etlichen Jahren von Liebler und Huber (2004) ebenfalls in dieser Zeitschrift abgehandelt.

Die Vorbereitung auf eine Gruppe hat im Wesentlichen die folgenden Ziele:

- Schaffen einer Basis für eine gute

(dauerhafte) therapeutische Arbeitsbeziehung,

- Abbau anfänglicher Ängste und Fehlvorstellungen, die Menschen im Hinblick auf Gruppen zeigen (s. oben),

- Vermitteln von Informationen über die Arbeitsweise einer Gruppe, die es Patienten erleichtern kann, der Teilnahme an einer Gruppentherapie auch aktiv zuzustimmen,

- Schaffen eines Konsenses über die Ziele der Therapie für den Einzelnen, Sichern der Zusammenarbeit und des Rahmens, aber auch Klären der Fragen, Probleme und Aufgaben, wenn dies möglicherweise in der Gruppe nicht erfolgen kann.

- Schließlich dient die Aufklärung auch der Vorhersage von Problemen, wenn etwa spezifische Befürchtungen auf der Patientenseite deutlich werden, die im Therapieverlauf umso rascher und effektiver aufgegriffen werden können.

In der oben erwähnten CORE battery wurden Materialien und Methoden zur Vorbereitung auf eine Gruppentherapie gesammelt, die von unterschiedlichen Autoren stammen (z.B. Rebecca Mac Nair-Semands, K. Roy MacKenzie) und die unterschiedliche Perspektiven darstellen. Die Handouts, die in Form einer offiziellen und autorisierten Über- 


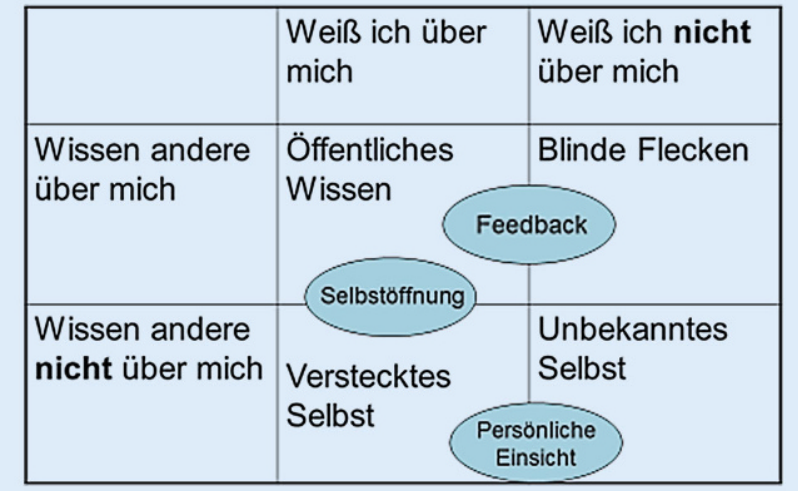

Abb. $1 \triangleleft$ Verschiedene Seiten des Selbst - das JohariFenster als Informationsmaterial für die Vorbereitung zur Gruppentherapie. (Nach MacKenzie 1997)

setzung in deutscher Sprache noch nicht vorliegen, umfassen Argumente und beispielhafte Formulierungen, mit denen Gruppentherapien gegenüber Patienten präsentiert werden können (z. B. „Neben den Gruppenleitern können Ihnen in der Gruppe auch die Mitpatienten sehr gut helfen. Alle Mitglieder werden dazu ermutigt, Beobachtungen und Eindrücke über die anderen zu äußern und in die Diskussion einzubringen"). In einem Handout beziehen sich die Aussagen auf die Möglichkeiten der optimalen Nutzung von Gruppen für die Patienten (z.B. „Wir ermutigen Sie sehr, über Ihre Erfahrungen und Gefühle zu sprechen. Sie werden am ehesten Fortschritte machen, wenn Sie sich selbst gestatten, Ihre wahren Gefühle und Reaktionen gegenüber anderen zu diskutieren und sich nicht scheuen, sich auch in Gebiete zu wagen, die für Sie emotional vielleicht unangenehm sind").

Ein ausführliches Handout aus der Feder von Roy MacKenzie (1997) greift v. a. den Informationsaspekt auf, die Fragen der Wirksamkeit und Wirkweise von Gruppen sowie eine ganze Reihe verbreiteter Mythen, die immer wieder mit Gruppeninterventionen verbunden werden. Auch hier werden sowohl entlastende Argumente (es ist ganz normal, sich in Gruppen auch ängstlich zu fühlen) und Anregungen zusammengefasst. Beispielsweise nutzt MacKenzie für die Aufklärung in diesem Handout das sog. Johari-Fenster (• Abb. 1, benannt nach den Urhebern, den amerikanischen Sozialpsychologen Joseph Luft und Harry Ingham). Dieses zeigt Patienten sehr plausibel, dass unterschiedliche Seiten der ei- genen Person für sich selbst und auch für andere in unterschiedlichem Maß sichtbar und deutlich werden, sowie dass wesentliche Mechanismen und Wirkfaktoren der Gruppentherapie (wie Selbstöffnung, Feedback und persönliche Einsicht) an diesen Kenntnissen über sich und andere etwas verändern können. Eine Aufgabe in der Gruppe kann sein, das mit „öffentlichem Wissen“ bezeichnete Feld zu vergrößern.

Wichtige Aspekte, die in der Vorbereitung thematisiert werden sollten, sind die Rahmenbedingungen und die Gruppenregeln (zu denen beispielsweise die Frage nach Kontakten zwischen den Sitzungen und v.a. die Vertraulichkeit gehören). Rahmenbedingungen und Gruppenregeln - auch hierfür gibt es ,,ein Muster" in der CORE battery - sollten am besten zu Beginn einer Gruppentherapie schriftlich von allen Gruppenteilnehmern zugesichert werden.

Im Zusammenhang mit der Information und Aufklärung ist zu betonen, dass die Materialien für die jeweiligen Gruppenangebote maßgeschneidert sein und alle relevanten Aspekte (theorie-, settingbezogen), die für ein spezifisches Vorgehen von Bedeutung sind, aufgreifen sollten.

Selbstverständlich gibt es viele Wege, die Information und Vorbereitung des Patienten zu gestalten. Persönliche Gespräche oder gar „psychoedukative Vorgruppen“" (Liebler und Huber 2004) sind sicherlich besonders sinnvoll, wobei diese durchaus durch schriftliche Informationen oder $u$. $U$. auch anderes mediales Informationsmaterial ergänzt werden können. Die US-amerikanischen
Beratungszentren an den Hochschulen, in denen extrem viele Gruppen durchgeführt werden und die untereinander sehr gut vernetzt sind, haben einen kurzen Informationsfilm kreiert, der im Internet erhältlich und zumindest geeignet ist, um die wesentlichen Aspekte und Inhalte der Gruppenvorbereitung noch einmal zusammenzufassen. Dieser Film findet sich unter folgendem Link: https:// amara.org/de/videos/Orcq0JQQ2bLF/ info/introduction-to-group-therapyby-darius-campinha-bacote/.

Im deutschsprachigen Raum hat sich eine kommerzielle Organisation unter dem Namen "Gruppenplatz" etabliert (www.gruppenplatz.de), auf deren Webportal (das dem Finden eines Gruppentherapieplatzes dienen soll) sich einige Kurzfilme finden, die durchaus aussagekräftig sind, indem sie Erfahrungen früherer Gruppenpatienten übersichtlich darstellen und zusammenfassen.

\section{M: Motivation und Auswahl}

In der CORE battery aus dem Jahr 2006 wurden 2 Instrumente näher beschrieben, die als „Screeningverfahren“ für die Auswahl und die Überprüfung der Motivation gegenüber Gruppentherapien genutzt werden können.

Es handelt sich um den „Group Selection Questionnaire“, der mittlerweile umbenannt wurde und unter dem Titel "Group Readiness Questionnaire" in der Literatur weiterverwendet wird (GRQ; Baker et al. 2013). Eine deutsche Übersetzung des GRQ liegt vor. Der Fragebogen beinhaltet 3 wesentliche Aspekte, nämlich die Erfassung der Erwartungen gegenüber Gruppen, die Fähigkeit, sich auf Gruppen einzulassen und eine Abschätzung von basalen sozialen Fertigkeiten. Der Fragebogen, der im Kontext eines Programms für Gruppeninterventionen mit traumaexponierten Jugendlichen entwickelt wurde, umfasst lediglich 19 Items und bedarf für das Ausfüllen kaum mehr als $5 \mathrm{~min}$. In einer Anwendung der deutschen Version von Löffler et al. (2007) hat sich der Fragebogen gut bewährt.

Sehr viel umfangreicher ist der „Group Therapy Questionnaire“ (GTQ; MacNair und Corazzini 1994), zu dem ein ausführ- 
liches Manual vorliegt (MacNair-Semands und Corazzini 1998). Der Fragebogen umfasst 44 Items, die sich auf 10 verschiedene Themengebiete verteilen, sowie eine zusätzliche Checkliste interpersonaler Merkmale, die wiederum aus 34 Items besteht. In dem Bogen sind Angaben zu Themen gebündelt, von denen aus der Forschung bekannt ist, dass sie für die Indikation zur Gruppentherapie von besonderer Bedeutung sind (z.B. negative Vorerfahrungen mit Gruppen, Ängste und Befürchtungen, negative Erwartungen und familiäre Rollen). Der Bogen wurde primär im Kontext studentischer Beratungsstellen in den USA entwickelt; Normwerte sind verfügbar. Allerdings ist zu reflektieren, welche Art von Gruppe für welche Patienten hilfreich und sinnvoll sein kann, bevor globale Kontraoder Pro-Indikationen auf der Basis dieses und anderer Screeninginstrumente gestellt werden.

\section{E: Evaluation und Wirksamkeits- messung}

In den letzten Jahrzehnten hat die Psychotherapieforschung immer deutlicher gezeigt, dass die Person des Therapeuten für die Vorhersage von Therapieergebnissen eine große Rolle spielt (Wampold et al. 2018). Dies ist eigentlich nicht verwunderlich, wurde aber lange Zeit in der Forschung nicht berücksichtigt.

Eine Fülle von Befunden zeigt, dass es sowohl im Hinblick auf bestimmte Ereignisse in der Therapie, die Qualität der therapeutischen Beziehung und das Ergebnis divergierende Sichtweisen gibt, mit einer gewissen Tendenz der Überschätzung von Therapieergebnissen aufseiten der Therapeuten (Cooper 2008) und der therapeutischen Allianz durch die Patienten (insbesondere in Abhängigkeit von der Symptomschwere; Tryon et al. 2007). Dazu passt der Befund, dass Psychotherapeuten ihre Qualitäten häufig überschätzen und sich die Mehrzahl den besten 10 ihrer Alterskohorten zurechnen.

Die Einschätzungen von therapeutischer Veränderung allein aus der Perspektive des Patienten und allein aus der Perspektive des Therapeuten weichen oft - auch in Gruppentherapien (Chapman et al. 2012) - sehr stark voneinander ab. Um diesen Bias zu kontrollieren, empfiehlt es sich, Verlaufsmessungen in Psychotherapien vorzunehmen, was selbstverständlich auch für die Gruppe gilt. Verlaufsmessungen bieten außerdem das Potenzial, den Behandelnden ein Feedback über Entwicklungen in der Gruppe zu geben, was in jüngster Zeit ein wesentliches Thema der patientenorientierten Gruppenpsychotherapieforschung geworden ist (Strauß et al. im vorliegenden Heft). Nach dem allgemeinen Wegfall der Antragspflicht in der Richtlinienpsychotherapie ist es nicht unwahrscheinlich, dass auch „offiziell“ ein Instrument zur Verlaufskontrolle für die Sicherung der Qualität der Psychotherapie zum Einsatz kommen wird.

Bezüglich der Empfehlung von Instrumenten zur Erfassung der Veränderung von Symptomen oder Zuständen bzw. dem Ergebnis-Monitoring kann zum einen auf die erwähnte CORE battery verwiesen werden: Dort werden exemplarisch 5 Methoden dargestellt, die sich - keineswegs gruppenspezifisch - auf die breite Erfassung von Symptomen beziehen:

- das Outcome Questionnaire (OQ-45) bzw. das Äquivalent für Jugendliche (Y-OQ),

- das Inventar zur Erfassung interpersonaler Probleme (IIP), das im deutschen Sprachraum bereits recht verbreitet ist,

- die Rosenberg-Selbstwertskala, die ebenfalls im deutschen Sprachraum verfügbar ist,

- der Gruppenbewertungsbogen von Froese bzw. Hess (1996), ein kurzes Instrument zur direkten Bewertung des subjektiven Nutzens von Gruppentherapien (Strauß et al. 1996) und schließlich

- die Erfassung von spezifischen Therapiezielen und deren Skalierung nach einer Methode von Battle et al. (1996).

Die Auswahl der Instrumente für die Veränderungsmessung in der CORE battery ist vermutlich etwas nordamerikanisch geprägt. Selbstverständlich sind alle im deutschen Sprachraum gängigen
Methoden (Symptom-Checkliste [SCL90], Beck-Depressions-Inventar [BSI], Patient Health Questionnaire [PHQ] etc.) auch geeignet.

Darüber hinaus empfiehlt es sich Instrumente einzusetzen, die sich auf spezifische Störungsbilder bzw. Diagnosen beziehen und eine diesbezügliche Veränderungsmessung erlauben. Hier$\mathrm{zu}$ zählen beispielsweise das BDI im Zusammenhang mit depressiven Störungen, Angstskalen, Skalen, die spezifisches Verhalten erfassen, oder die YaleBrown Obsessive Compulsive Scale, die international in Studien zur Gruppentherapie von Zwangsstörungen als „KernOutcome-Instrument" eingesetzt wird (Schwartze et al. 2016). Bezüglich weiterer störungsspezifischer Instrumente können metaanalytische Zusammenfassungen von Therapiestudien aus dem Gruppenkontext inspirieren, auf die in dem Beitrag von Strauß et al. (im vorliegenden Heft) eingegangen wurde.

In jüngster Zeit wurde in der Psychotherapie zunehmend deutlich, dass sich bei der Abschätzung von Wirkungen der Psychotherapie nicht allein auf die positiven Veränderungen und Symptomreduktionen verlassen werden kann. Auch Nebenwirkungen und unerwünschte Wirkungen von Psychotherapie müssen gesondert betrachtet werden. Diese Debatte wird häufig unter dem Stichwort der Nebenwirkungsforschung in der Psychotherapie geführt (Linden und Strauß 2018). Entsprechend haben sich in den jüngsten Jahren einige Methodenentwicklungen ergeben, die spezifisch auf Nebenwirkungen, negative Effekte, adverse Effekte und problematische Entwicklungen in der Psychotherapie und in der Gruppentherapie richten. $\mathrm{Zu}$ Letzterem gehört der „Fragebogen zu Nebenwirkungen in der Gruppentherapie und unerwünschten Gruppenerfahrungen“ (NUGE), der im Kontext stationärer Gruppentherapieangebote entwickelt, mittlerweile aber auch als in Ausbildungsambulanzen generalisierbar beschrieben wurde. Der "NUGE 24" (Strauß und Drobinskaya 2018) fokussiert auf das von Strauß et al. (im vorliegenden Heft) beschriebene Modell zu Einflussfaktoren auf das Ergebnis von Gruppenpsycho- 
Tab. 2 Operationale Aspekte und Definitionen der gruppenleiterbezogenen Inhaltsbereiche in der Group Psychotherapy Intervention Rating Scale (GPIRS). (Nach Chapman et al. 2010)

\begin{tabular}{l|l}
\hline $\begin{array}{l}\text { GPIRS- } \\
\text { Bereich }\end{array}$ & $\begin{array}{l}\text { Operationale A } \\
\text { Interventionen }\end{array}$ \\
\hline $\begin{array}{l}\text { Strukturierung } \\
\text { der Gruppe }\end{array}$ & $\begin{array}{l}\text { Identifikation } \\
\text { Erklärung } \\
\end{array}$ \\
& Erleichterung \\
\hline
\end{tabular}

\begin{tabular}{|c|c|c|}
\hline $\begin{array}{l}\text { Verbale Inter- } \\
\text { aktion }\end{array}$ & $\begin{array}{l}\text { Modellfunktion als Leiter } \\
\text { Verbale Modellfunktion } \\
\text { Selbstöffnung } \\
\text { Feedback }\end{array}$ & $\begin{array}{l}\text { Interventionen, die angemessene Verbalisierun- } \\
\text { gen und Interaktionsstile (einschl. Feedback und } \\
\text { Selbstoffenbarung) fördern }\end{array}$ \\
\hline $\begin{array}{l}\text { Emotionales } \\
\text { Klima }\end{array}$ & $\begin{array}{l}\text { Balance zwischen Unterstüt- } \\
\text { zung und Konfrontation } \\
\text { Vermeiden von Feindselig- } \\
\text { keit } \\
\text { Unterstützung } \\
\text { Beteiligung von Mitgliedern } \\
\text { Prävention von problemati- } \\
\text { schen Interaktionen }\end{array}$ & $\begin{array}{l}\text { Interventionen, die den Mitgliedern helfen, } \\
\text { Emotionen in einer kathartischen und konstruk- } \\
\text { tiven Weise auszudrücken (dazu gehören the- } \\
\text { rapeutische Interventionen wie Unterstützung, } \\
\text { Konfrontation, Offenheit und Wärme) }\end{array}$ \\
\hline
\end{tabular}

therapien. Er differenziert letztendlich 4 Skalen, die mit den Bezeichnungen „Belastung durch die Gruppe“, „Belastung durch Therapeut/-in“, „Belastung durch andere Patienten“ und „persönliche Überforderung“ versehen wurden. In einer Pilotstudie zeigte sich die Eignung des NUGE 24, Nebenwirkungen und unerwünschte Erfahrungen differenziert (z.B. nach Setting und nach gruppentherapeutischen Theorieansatz) zu erfassen.

\section{L: Leiterstil und Leiterverhalten}

Die Forschung zur Person des Gruppenleiters/der Gruppenleiterin und entsprechende Instrumente sind in der Gruppenliteratur noch vergleichsweise schwach vertreten (was der Lage in der Psychotherapieforschung allgemein entspricht). Es gibt einige nützliche Modelle und Beschreibungen bezüglich der Aufgaben einer Gruppenleitung. Zu nennen sind z.B. Liebermans Modell der wesentlichen Interventionsstrategien in der Gruppe (Lieberman et al. 1973): exekutive Funktionen, Fürsorge, emotionale Stimulation und Bedeutungszuweisung oder eine Abwandlung des Situational Leadership Model von Hersey und Blanchard. Dieses skaliert das Leiterverhalten anhand der Dimensionen „Supportivität“ und „Direktivität" sowie in Abhängigkeit von der Grundmotivation und der Eignung von Patienten für die Gruppe (Strauß 2018, 2020).

Instrumente zur Erfassung des Leiterverhaltens sind noch relativ rar und sollten insbesondere im Hinblick auf methoden- und auch settingübergreifende Anwendbarkeit überprüft werden, zumal Gruppenerfahrungen sich potenziell in unterschiedlich theoretisch begründeten Gruppenpsychotherapien voneinander unterscheiden können (Watzke et al. 2004).

Ein Instrument, das ein Fremd-Rating der Therapeuteninterventionen umfasst und dazu dienen kann, differenzielle Aspekte des Leiterverhaltens abzubilden, wurde ursprünglich von einer holländischen Arbeitsgruppe um Wim Trijsburg als Group Psychotherapy Intervention Rating Scale (GPIRS; Sternburg und Trijsburg 2005) entwickelt. Die GPIRS wurde empirisch auf der Basis von Beobachtungen und theoretischen Konzepten bezüglich der Frage konstruiert, wie eine Gruppenleitung dazu beitragen kann, dass sich Kohäsion etabliert und damit eine Arbeitsfähigkeit der Gruppe herstellt. Die Rating-Skala, die von Chapman et al. (2010) validiert wurde, umfasst Items, die sich auf die Strukturierung der Gruppe, auf verbale Interventionen, einschließlich gezielter Selbstoffenbarungen vonseiten der Gruppenleiter, und Feedback beziehen. Des Weiteren wird eine Reihe von Aspekten und Skills, die im Zu- sammenhang mit der Kreation und Aufrechterhaltung eines therapeutischen Arbeitsklimas verbunden sind, analysiert. Hierzu zählt beispielsweise die Tatsache, dass der Gruppenleiter dafür sorgt, konfrontative und emotional unterstützende Äußerungen in ein Gleichgewicht zu bringen (• Tab. 2).

\section{D: Dokumentation}

Für die Dokumentation von Gruppensitzungen gab es bislang kaum sinnvolle Ansätze, abgesehen von "handgestrickten "Protokollbogen, die in unterschiedlichen Einrichtungen in unterschiedlicher Form angewandt werden.

Die Ausbildung zum Gruppentherapeuten erfolgt nach wie vor unzureichend (Strauß et al. 2012), und viele Menschen, die gruppentherapeutisches Arbeiten lernen wollen, sind (insbesondere in der Psychotherapieausbildung, vermutlich auch künftig in der Psychotherapieweiterbildung) auf sich gestellt. Inspiriert durch diese Erkenntnisse wurde in der eigenen Arbeitsgruppe die operationale Basisdokumentation für Gruppentherapie (OBG) entwickelt, die bereits ausführlich beschrieben wurde (Schubert et al. 2016; Degott et al. 2017). Die OBG orientiert sich an dem Modell, das verschiedene Einflussfaktoren auf den Effekt von Gruppentherapien unterscheidet sowie zwischen der anatomischen Struktur einer Gruppe und physiologischen Gruppenprozessen differenziert (Schubert et al. 2016), und wurde in einer Praxisstudie erprobt (Degott et al. 2017).

Für diese Modellkomponenten wurden Dokumentationsitems gebildet, die in einem ausführlichen Manual beschrieben sind (Schubert et al. 2016). Diese Items umfassen beispielsweise die inhaltlichen Schwerpunkte einer Sitzung, Fragen der Subgruppenbildung, der Normierung einer Gruppe und der Gruppenentwicklung, Rahmenbedingungen, die vom Gruppenleiter festgelegt werden können, und die Bedeutung sozialpsychologischer Prozesse wie Konflikte und den Umgang damit. Auch die Einschätzung der therapeutischen Faktoren von Gruppentherapien hat in der Dokumentation ihren Platz, 
ebenso wie die oben genannten Modelle des Gruppenleiterverhaltens bzw. -stils.

Eine erste Praxisstudie von Degott et al. (2017) hat ergeben, dass die Dokumentation von vielen Gruppentherapeuten als sehr hilfreich erlebt wird, allerdings für die praktische Routine etwas zu umfangreich ist (nicht so für Supervisionszwecke). Deshalb wird derzeit eine etwas verkürzte Form entwickelt, mit der im ersten Praxisversuch (Frick 2019) die Zeit für das Ausfüllen der Dokumentation von ca. 16 auf ca. $8 \mathrm{~min}$ reduziert wurde, was in der Praxis deutlich zumutbarer erscheint.

\section{A: Allianz und andere Prozessmerkmale}

Wie in dem Beitrag von Strauß et al. im vorliegenden Heft gezeigt, existiert die mit Abstand meiste Literatur aus der Prozessforschung von Gruppenpsychotherapien zu den Konstrukten der therapeutischen Allianz (Alldredge et al. im Druck) und Kohäsion (Burlingame et al. 2018).

Prozessmerkmale werden oft als zugrunde liegende Veränderungsmechanismen in der Psychotherapie begriffen, obwohl ihre Evidenz, insbesondere die Kausalität dieser Mechanismen betreffend, noch begrenzt ist.

In neuen Studien wurden als die am häufigsten genutzten Instrumente zur Erfassung von Prozessaspekten in Gruppen das Working Alliance Inventar(WAI), die Empathie-Skala (ES), die Kurzform des Gruppenklimafragebogens (GCQ) sowie die Kohäsionsskala aus dem Therapeutic Factors Inventory (TFI) identifiziert. Diese wurden sodann in empirischen Untersuchungen (z. B. Bormann und Strauß 2007) akribischen Zusammenhangsanalysen unterzogen. Die Messinstrumente formieren 3 wesentliche Komponenten der Gruppentherapiebeziehungserfahrung, nämlich die Erfahrung positiver Beziehungen auf eher emotionaler Ebene, die positiven Arbeitsbeziehungen und negative Faktoren. Bei Letzteren handelt es sich um Faktoren, die mit den beiden anderen möglicherweise interferieren, sich aber nicht notwendigerweise ausschließen, zumal die verschiedenen Konstrukte auf unterschiedlichen Per- spektiven angesiedelt sein können (allen voran der Beziehung zwischen den Gruppenmitgliedern und dem Gruppenleiter bzw. der Beziehung der Gruppenmitglieder zur Gruppe als Ganzes). Das aus diesen Analysen entstandene Group Questionnaire (GQ) ist seit längerer Zeit in deutscher Sprache verfügbar (Bormann et al. 2011). Die Faktorenstruktur wurde mehrfach (auch in mehreren Ländern) repliziert.

Neben dem GQ wurden die einzelnen oben erwähnten Messinstrumente als potenzielle Bestandteile einer CORE battery beschrieben. Zusätzlich noch zur Erfassung der Bindung an den Therapeuten die Cohesion to the Therapist Scale (CTS) und ein Fragebogen zur Erfassung kritischer Ereignisse (Critical Incident Questionnaire) in die CORE battery aufgenommen.

Im deutschsprachigen Raum wurden in jüngerer Zeit weitere Instrumente zur Prozessforschung in der Gruppenpsychotherapie entwickelt (Vogel et al. 2016), z.B. ein Fragebogen zur Erfassung der Wirkfaktoren von Gruppen aus der therapeutischen Perspektive (Winter et al. im Druck). Ähnlich wie in der Einzeltherapie sind die Konstrukte, die als Prozessmerkmale in der Gruppe diskutiert werden, auch potenzielle Veränderungsmechanismen (CritsChristoph et al. 2013), wenngleich eine entsprechende Differenzierung im Gruppenkontext noch nicht sehr weit fortgeschritten ist.

\section{Fazit für die Praxis}

- Das Konzept „IMELDA“ hat unterschiedliche Aspekte des gruppentherapeutischen Arbeitens im Blick (I: Information und Vorbereitung, M: Motivation und Auswahl, E: Evaluation und Wirksamkeitsmessung, $L$ : Leiterstil und Leiterverhalten, $D$ : Dokumentation sowie A: Allianz und andere Prozessmerkmale).

- Es werden Materialien und Methoden zur Verfügung gestellt, die es gruppentherapeutisch Tätigen erleichtern sollen, Gruppenteilnehmer adäquat vorzubereiten und zu informieren, sie einem ersten Screening zu unterziehen sowie im Gruppenverlauf
Veränderungen im Prozess und in der Symptomatik bzw. Belastung der Patienten aus unterschiedlichen Perspektiven zu erfassen.

- Alles zusammen sollte v.a. im institutionellen Kontext dazu beitragen, dass auch die Ausbildungsqualität im Zusammenhang mit gruppentherapeutischer Tätigkeit verbessert wird.

- Wenn die gesundheitspolitische und wissenschaftlich gut begründete Absicht, die Gruppentherapien zu fördern, realisiert werden soll, brauchen Gruppenpsychotherapeuten nicht nur strukturelle und ideelle, sondern auch die konkrete Unterstützung in Form des Materials, das IMELDA bietet.

\section{Korrespondenzadresse}

\section{Prof. Dr. Bernhard Strauß}

Institut für Psychosoziale Medizin und Psychotherapie, Universitätsklinikum Jena

Stoystr. 3, 07740 Jena, Deutschland

Bernhard.Strauss@med.uni-jena.de

Funding. Open Access funding provided by Projekt DEAL.

\section{Einhaltung ethischer Richtlinien}

Interessenkonflikt. B. Strauß gibt an, dass kein Interessenkonflikt besteht.

Für diesen Beitrag wurden vom Autor keine Studien an Menschen oder Tieren durchgeführt. Für die aufgeführten Studien gelten die jeweils dort angegebenen ethischen Richtlinien.

Open Access. Dieser Artikel wird unter der Creative Commons Namensnennung 4.0 International Lizenz veröffentlicht, welche die Nutzung, Vervielfältigung, Bearbeitung, Verbreitung und Wiedergabe in jeglichem Medium und Format erlaubt, sofern Sie den/die ursprünglichen Autor(en) und die Quelle ordnungsgemäß nennen, einen Link zur Creative Commons Lizenz beifügen und angeben, ob Änderungen vorgenommen wurden.

Die in diesem Artikel enthaltenen Bilder und sonstiges Drittmaterial unterliegen ebenfalls der genannten Creative Commons Lizenz, sofern sich aus der Abbildungslegende nichts anderes ergibt. Sofern das betreffende Material nicht unter der genannten Creative Commons Lizenz steht und die betreffende Handlung nicht nach gesetzlichen Vorschriften erlaubt ist, ist für die oben aufgeführten Weiterverwendungen des Materials die Einwilligung des jeweiligen Rechteinhabers einzuholen. 
Weitere Details zur Lizenz entnehmen Sie bitte der Lizenzinformation auf http://creativecommons.org/ licenses/by/4.0/deed.de.

\section{Literatur}

Alldredge C, Rosendahl J, Yang C, Burlingame GM (im Druck) Alliance in group therapy: A metaanalysis. Psychotherapy

BakerE, Burlingame GM, Cox M, Jonathan C, Beecher M, Gleave E, Robert L (2013) The group readiness questionnaire: a convergent validity analysis. Group Dyn 17(4):299-314

Battle CC, Imber SD, Hoehn-Saric R et al (1996) Targe complaints as criteria for improvement. Am J Psychother 20:184-192

Bormann B, Strauß B (2007) Gruppenklima, Kohäsion, Allianz und Empathie als Komponenten der therapeutischen Beziehung in Gruppenpsychotherapien - Überprüfung eines Mehrebenenmodells. Gruppenpsychother Gruppendyn 43:1-20

Bormann B, Burlingame GM, Strauß B (2011) Der Gruppenfragebogen: Die deutsche Version des Group Questionnaire (GQ-D) - Ein Instrumentzur Messung von therapeutischen Beziehungen in der Gruppenpsychotherapie. Psychotherapeut 56(4):297-309

Bundespsychotherapeutenkammer (2018) Ein Jahr nach der Reform der Psychotherapierichtlinie. BPtK, Berlin. https://www.bptk.de/wpcontent/uploads/2019/01/20180411_bptk studie_wartezeiten_2018.pdf

Bundesregierung Koalitionsvertrag von CDU, CSU und SPD (2014) Deutschlands Zukunft gestalten. Bundestagsdrucksache. Bundesregierung Koalitionsvertrag von CDU, CSU und SPD, Berlin

Burlingame GM et al (2006) CORE-Battery-Revised: An assessment tool kit for promoting optimal group selection, process and outcome. AGPA, New York

Burlingame GM, MacKenzie KR, Strauss B (2004) Small group treatment: evidence for effectiveness and mechanisms of change. In: Lambert MJ (Hrsg) Bergin \& Garfield's handbook of psychotherapy and behavior change, 5. Aufl. Wiley, New York, S647-696

Burlingame GM, McClendon DT, Yang C (2018) Cohesion in group therapy: a meta-analysis. Psychotherapy 55(4):384-398

Chapman CL, Baker E, Porter G, Thayer S, Burlingame GM (2010) Rating group therapist interventions: the validation of the group psychotherapy intervention rating scale. Group Dyn 14:15-31

Chapman $\mathrm{CL}$, Burlingame GM, Gleave R, Rees F, Beecher M, Porter GS (2012) Clinical prediction in group psychotherapy. Psychother Res 22(6):673-681

Cooper M (2008) Essential research findings in counsellingand psychotherapy.SAGE, Thousand Oaks

Crits-Christoph P, Connolly MB, Mukherjee D (2013) Psychotherapy process outcome research. In: Lambert MJ (Hrsg) Bergin \& Garfield's handbook of psychotherapy and behavior change, 5. Aufl. Wiley, New York, S298-340

Degott N, Schubert T, Strauß B (2017) Operationalisierte Basisdokumentation von Gruppenpsychotherapien - Ergebnisse einer ersten Erprobung. Psychotherapeut 62:537-546
Frick A (2019) Erstellung einer Kurzform der OBG. Masterthese. Institut für Psychologie, Friedrich Schiller Universität, Jena

Hess H (1996) Zwei Verfahren zur einschätzung der Wirksamkeit von Gruppentherapie. In: Strauß B Eckert J, Tschuschke V (Hrsg) Methoden der empirischen Gruppentherapieforschung - ein Handbuch. Westdeutscher Verlag, Opladen, S142-158

Lieberman MA, Yalom ID, Miles MB (1973) Encounter groups: First facts. Basic Books, New York

Liebler A, Huber TJ (2004) Die Therapievorbereitungsgruppe. Psychotherapeut 49:272-276

Linden M, Strauß B (2018) Risiken und Nebenwirkungen von Psychotherapie, 2. Aufl. Wissenschaftlich Medizinischer Verlag, Berlin

Löffler J, Bormann B, Burlingame GM, Strauß B (2007) Auswahl von Patient(inn)en für Gruppenpsychotherapien - Validierung der deutschen Version des Group Selection Questionnaire (GSQ). ZPsychiatr Psychol Psychother 55:75-86

MacKenzie KR (1997) Time-managed group psychotherapy. APA Press, Washington

MacKenzie KR, Dies R (1982) AGPA CORE-Battery. AGPA, New York

MacNair RR, Corazzini J (1994) Client factors influencing group therapy dropout. Psychotherapy 31:352-361

MacNair-Semands R, Corazzini J (1998) Manual for the group therapy questionnaire (GTQ). Virginia Commonwealth University, Counseling Services, Charlotte

Schubert T, Degott N, Strauß B (2016) Entwicklung eines Systems zur operationalisierten Basisdokumentation von Gruppenpsychotherapien (OBG): 1. Grundlagen und Beschreibung des Systems. Psychotherapeut 61:376-383

Schwartze D, Barkowski S, Burlingame GM, Strauss B Rosendahl J (2016) Efficacy of group psychotherapy for obsessive-compulsive disorder: A meta-analysis of randomized controlled trials. JObsessive Compuls Relat Disord 10:49-61

Sternburg S, Trijsburg W (2005) The relationship between therapeutic interventions and therapeutic outcome. University of Amsterdam, Amsterdam

Strauß B (2018) Formale Veränderungstheorien und Gruppenleitung. In: Strauß B, Mattke D (Hrsg) Gruppenpsychotherapie - Lehrbuch für die Praxis, 2. Aufl. Springer, Heidelberg, S 181-190

StraußB (2020) Grundlagen der Gruppenpsychotherapie. Kohlhammer, Stuttgart

Strauß B, Drobinskaya A (2018) Erste Erfahrungen mit dem „Fragebogen zu Nebenwirkungen in der Gruppentherapie und unerwünschten Gruppenerfahrungen" (NUGE 24). Psychother Psychosom Med Psychol 68:437-442

Strauß B, Burlingame GM, Bormann B (2008) Using the CORE-R battery in group psychotherapy. J Clin Psychol 11(64):1225-1237

Strauß B, Eckert J, Tschuschke V (Hrsg) (1996) Methoden zur empirischen Forschung in der Gruppenpsychotherapie - Ein Handbuch. Westdeutscher Verlag, Opladen

StraußB, Schreiber-Willnow K, Kruse J, Schattenburg L, Seidler KP, Fischer Th, Papenhausen R, Möller E, Dobersch J, Wünsch-Leiteritz W, Leiteritz A, Huber Th, Kriebel R, Liebler A, Mattke D, Weber R, Bormann B (2012) Ausbildungshintergrund, Alltagspraxis und Weiterbildungsbedarf von Gruppenpsychotherapeuten in der stationären Psychotherapie - Ergebnisse einer Umfrage. ZPsychosom Med Psychother 58:394-408
Tryon GS, Blackwell SC, Hammel EF (2007) A meta-analytic examination of client-therapist perspectives of working alliance. Psychother Res 17:629-642

Vogel E, BlanckP, Bents H, Mander J(2016) Wirkfaktoren in der Gruppentherapie: Entwicklung und Validierung eines Fragebogens. Z Psychosom MedPsychother 66(5):170-179

Wampold B, Imel Z, Flückiger C (2018) Die große Psychotherapie Debatte. Hogrefe, Göttingen

Watzke B, Scheel S, Bauer C (2004) Differenzielle Gruppenerfahrungen in psychoanalytisch und verhaltenstherapeutischen begründeten Gruppenpsychotherapien. Psychother Psychosom Med Psychol 54:348-357

Winter F, Aguilar-Raab C, Blanck P, Schaller G, Vogel E, Munder J (im Druck) Wirkfaktoren der Gruppentherapie: Fragebogen zur Messung der therapeutischen Perspektive. Psychother Psyhc Med. https://doi.org/10.1055/a-1012-2078 